\title{
Stochastic user equilibrium traffic assignment with equilibrated parking search routes
}

Adam J. Pel ${ }^{\text {a, }}$, Emmanouil Chaniotakis ${ }^{b}$

a Department of Transport and Planning, Delft University of Technology, The Netherlands

${ }^{\mathrm{b}}$ Department of Civil, Geo and Environmental Engineering, Technical University of Munich, Germany

*Corresponding author: a.j.pel@tudelft.nl

\section{Highlights:}

- We define and formulate the concept of parking search routes (PSR) accounting for parking probabilities.

- We propose a queuing model to compute endogenous parking probabilities accounting for PSRs and search times.

- We define and formulate the stochastic user equilibrium (SUE) assignment with equilibrated PSRs (PSR-SUE).

- We propose a method for PSR choice set generation, and a solution algorithm to solve the PSR-SUE problem.

- We demonstrate the PSR-SUE model properties, and analyze the model application to a real-life setting.

\begin{abstract}
:
In this paper we define and formulate the concept of parking search routes (PSR) where a driver visits a sequence of parking locations until the first vacant parking spot is found and in doing so may account for (expected) parking probabilities. From there we define and formulate the stochastic user equilibrium (SUE) traffic assignment in which no driver, by unilaterally changing its PSR, can lower its perceived expected generalized costs. Recognizing the interdependency between PSR flows, travel times and parking probabilities, we propose a queuing model in order to compute endogenous parking probabilities accounting for these factors as well as maximum admissible search times. To solve the SUE assignment with equilibrated PSR we propose a solution algorithm, including a method for PSR choice set generation. The model is implemented and applied both to a number of experimental cases to verify its properties and to a real-life setting to illustrate its usefulness in parking-related studies.
\end{abstract}

\section{Keywords:}

Parking; Traffic assignment; Stochastic user equilibrium; Choice modeling; Queuing theory 


\section{Introduction}

Parking is an essential component in every car trip especially in the urban context, and as such has been studied regarding, for example, how it affects mode choice, location accessibility, and network performance due to cruising traffic. In particular considering cruising traffic, various studies have quantified this with estimates of about $30 \%$ of the urban traffic flows being cruising traffic (e.g., Shoup, 2006, and Van Ommeren et al., 2012) and of about $30-50 \%$ of the travel time within the city being spent on searching for a parking spot (e.g., Bonsall and Palmer, 2004, and Tang et al., 2014).

Apart from the sheer amount of cruising traffic, parking in the urban context is also an interesting topic for research due to fast developments nowadays in the parking system. In more and more cities worldwide, including the majority of cities in the Netherlands, on-street paid parking is regulated by registration of the car number plate, instead of the traditional pay-and-display system where a parking ticket is bought. The main reason for implementing this payment system is that it enables automated parking control, using a special control car with mounted automatic number plate recognition camera that is connected with the back office system to check for any non-registered parked cars. This online payment system has led to the introduction of several smartphone applications where users no longer need to pay in advance for a pre-specified amount of parking time, but simply register their car upon arrival at a parking location and subsequently unregister upon departure, thus only paying for the parking time actually used. Given that parked cars are now registered, this means that there is real-time information on the occupancy at parking locations. This information was already available for off-street parking, but is generally only displayed at roadside panels that are typically located at motorway exits and along the urban ring road for purposes of parking route guidance. Now with information on onstreet parking occupancies as well and with smartphone applications, this parking occupancy information may soon become available to drivers everywhere and in realtime. Furthermore, this may enable parking reservation in advance. Such a reservation system is not yet available for public parking space, although a few of the Dutch smartphone applications do already offer this service for private parking space offered by, for example, companies and hotels.

Earlier studies on parking location choices of drivers show that this predominantly depends on the factors of access time to parking location, parking fees, walking distance to final destination, and a number of socio-economic characteristics of the driver. In a recent stated preference survey by the authors (Chaniotakis and Pel, 2015) we show that uncertain parking availability ranks as second most important factor in determining drivers' parking location decisions, and is only dominated by parking fees. This is relevant because the parking availability and associated search time is evidently precisely what will be affected by the aforementioned new technologies, while the other factors will remain mostly unaffected. Furthermore, earlier studies on parking information and guidance systems show that the effects hereof are rather 
limited when the parking information provided via roadside panels tended to be outdated and unreliable, especially in more congested circumstances (see e.g., Waterson et al., 2001, and Geng and Cassandras, 2012).

In this paper we propose a model for the dynamic traffic assignment problem that incorporates drivers' parking search routes in a way that allows to simulate the effects of uncertain parking availability and parking reservation, and thus enables evaluating various parking information technologies and policies. According to Martens and Benenson (2008) this model would be classified as a spatially explicit parking model, as opposed to a spatially implicit model that only considers parking location without accounting for the traffic network effects. Examples of spatially implicit models are: the bottleneck model proposed by Yang et al. (2013) that is used by Liu et al. (2016) to analyze the effects of parking pricing and regulation on departure times in the morning commute; the network fundamental diagram model proposed by Geroliminis (2015) and Liu and Geroliminis (2016) that is used to analyze the relationships between time-varying tolls, departure times, and cruising times of morning commute traffic; and the probabilistic system dynamics model proposed by Cao and Menendez (2015) that is used to analyze the interactions between traffic flows, parking supply, and cruising times. On the other hand, considering spatially explicit parking models, also various approaches have been proposed in the literature. For example, a number of simulation models have been proposed to describe parking traffic, in particular the on-street search process where drivers cruise in a myopic semi-random manner searching for a vacant parking spot (e.g., Kaplan and Bekhor, 2011, Van der Waerden, 2012, Guo et al., 2013, and Boyles et al., 2014). Along a similar line of reasoning, a number of agent-based simulation models have been appended with decision rules for parking choices (e.g., Benenson et al., 2008, and Waraich and Axhausen, 2012). Other studies have considered strategic parking search behavior within an equilibrium context and use network assignment models. For example, Bifulco (1993) solves the stochastic user equilibrium assignment where route costs include parking search costs that are approximated as a function of parking occupancy. Lam et al. (2006) solve the traffic assignment problem with departure time and parking location choice where parking availability is approximated via a BPR-like cost function. Li et al. (2008) solve the traffic assignment problem under the assumption of time-dependent Normaldistributed uncertain travel times and parking search times in order to investigate the impact on network reliability. Leurent and Boujnah (2014) solve the static user equilibrium assignment with route and parking location choice, where drivers divert to other parking locations when not being able to find a vacant parking spot, such that parking search routes emerge. Boyles et al. (2015) model strategic parking routes within the cell transmission model by incorporating parking search policies defined in terms of stochastic decision processes. Their approach computes the user equilibrium traffic assignment that minimizes travel times (including driving time and walking time towards the destination) with endogenous parking availabilities.

All of these model studies in one way or another account for uncertain parking availability and the parking search process, whereas the majority of parking models 
(used to study e.g., parking pricing and regulation) would typically model parking availability as deterministic. Although these models may certainly be applicable for specific studies, they also have their limitations within the context of evaluating the impact of all kinds of parking-oriented traffic information and management strategies (such as parking information systems, parking guidance systems, parking reservation systems, and all sorts of parking policies targeted towards reducing cruising traffic). The latter scope of model applications requires (1) a spatially explicit parking model as part of a traffic assignment model, (2) including drivers' choice behavior (pre-trip and on-trip) among parking locations, and the effect of probabilistic parking availability and search times, (3) as well as time dynamics of traffic flows and parking availabilities, (4) and where the interdependencies between parking search route flows, travel times, and parking probabilities are endogenously determined in a rigorous manner. To the best of the authors' knowledge no model to date meets all of these requirements.

In this paper we propose a model that does meet these requirements. It does so by distinguishing itself particularly in two ways. First, our model has a clear theoretical foundation where parking search route choice follows Random Utility Maximization choice theory, and the (stochastic) user equilibrium assignment with equilibrated parking search routes is a generalization of the Wardrop equilibrium concept. Second, the rigorous model formulation includes the interdependencies between parking search route flows, travel times, and parking probabilities, and hence these three factors are all endogenously determined. As such, the model adheres to general requirements for (traffic assignment) planning models.

The contributions of the paper are: (1) we define and formulate the concept of parking search routes (PSR) that drivers may follow in order to find a vacant parking spot while accounting for parking probabilities; (2) we propose a queuing model to compute endogenous parking probabilities accounting for PSRs and search times; (3) we define and formulate the stochastic user equilibrium (SUE) assignment with equilibrated PSRs (PSR-SUE); (4) we propose a method for PSR choice set generation, and a solution algorithm to solve the PSR-SUE problem; (5) we demonstrate the PSR-SUE model properties, and analyze the model application to a real-life setting.

The paper is structured as follows. In Section 2 we define and formulate parking search routes and the stochastic user equilibrium assignment with equilibrated parking search routes, as well as propose a solution algorithm for this problem. In Section 3 the model is applied to several example and real-life cases, and discussed with respect to model properties and some illustrative parking scenarios. In Section 4 we draw conclusions.

\section{PSR-SUE model formulation}

\subsection{Equilibrated parking search routes}

Recall that we are interested in drivers' parking locations and search routes. To this 
end, we propose the following definition.

Definition 1. Parking search route (PSR). A parking search route is a route starting at the driver's origin and sequentially connecting a number of parking locations which can be either (off-street) parking facilities or (on-street) parking areas. Hence a PSR consists of a sequence of route segments, where each segment connects a consecutive pair of parking locations, apart from the first segment that starts at the origin and ends at the first parking location to be visited. This way, a driver will follow a PSR and visit these parking locations until the first vacant parking spot is found (and the remainder of the PSR is aborted).

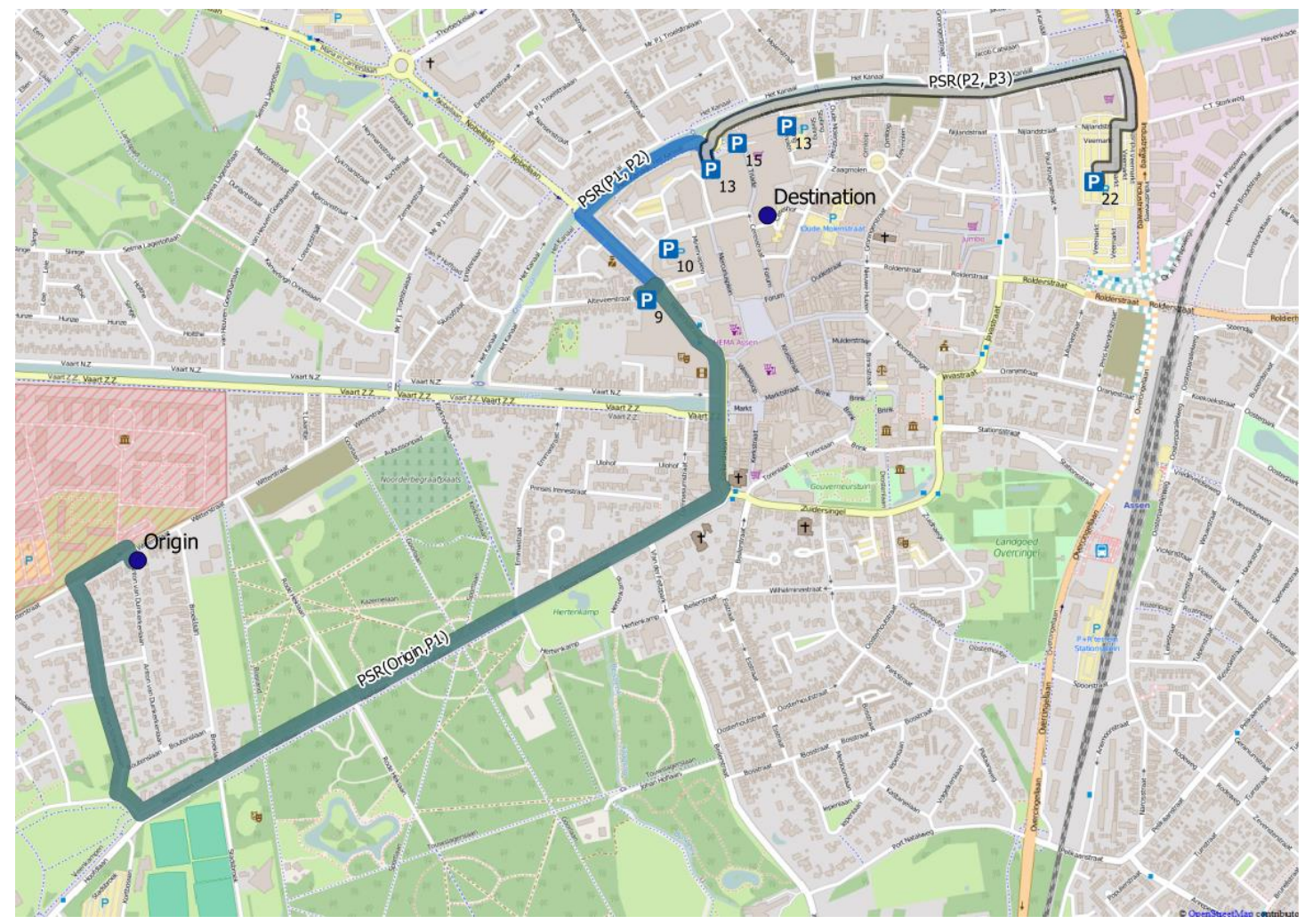

Fig. 1. Example of a parking search route (PSR) for an origin and a destination. The PSR consists of three route segments (highlighted), which start at the origin and sequentially connect three parking locations.

The concept of a parking search route is illustrated in Fig. 1. An example is shown of a parking search route that consists of three route segments, starts at the origin, and sequentially connects three parking locations that all lie in the proximity of the destination. Parking locations here refer to parking facilities or neighborhood areas where parking is provided. In the ensuing we consider the strategic choices of drivers regarding how they consecutively visit these parking locations in order to find a parking spot. This way, these strategic parking choices are complementary to the onstreet search process where drivers cruise in a myopic semi-random manner searching for a vacant parking spot within such a neighborhood area (and similarly there exist models that describe the search process for a vacant parking spot within an off-street 
parking facility, but this is beyond our scope as we are interested in how parking search traffic affects the on-street traffic conditions). Note that re-visiting parking locations within a parking search route is rather unlikely (although this is theoretically possible as parking probabilities are time-dependent), contrary to revisiting individual streets during the on-street search process within a neighborhood which is more behaviorally plausible. Finally, in the model application in section 3.2 we append our model describing parking search routes with the model by Kaplan and Bekhor (2011) describing the on-street search process.

This definition of a parking search route (PSR) allows us to define the stochastic user equilibrium (SUE) traffic assignment with equilibrated parking search routes. We propose the following definition.

Definition 2. Stochastic user equilibrium traffic assignment with equilibrated parking search routes (PSR-SUE). The stochastic user equilibrium traffic assignment with equilibrated parking search routes is the traffic flow assignment in which no driver, by unilaterally changing its PSR, can lower its perceived expected generalized costs.

Note that following the definition of a PSR the latter generalized costs naturally take into account (uncertain) parking availabilities. Hence, drivers, upon selecting their PSR, respond to expected probabilities of parking availability at the various parking locations. The PSR-SUE assignment is thus flexible enough to capture the typical urban setting, where drivers may be familiar with the average (day-to-day) probabilities of parking availability at the various parking locations (but are unaware of the actual (current day) availability status), as well as capture the situation in which a parking information or reservation system is employed, where these 'probabilities' would be close to binary.

Next, we formulate and solve the SUE with equilibrated PSR problem.

\subsection{PSR-SUE problem formulation}

\section{Flow assignment}

In line with the definition of the PSR-SUE problem, the PSR-SUE conditions can be formulated as follows.

Definition 3. PSR-SUE conditions. Under the PSR-SUE assignment, for any origindestination flow, the expected perceived generalized costs for all used PSR are equal, and greater than or equal to the expected perceived generalized costs for any unused PSR.

For reference with respect to denotation, Table 5 in Appendix A provides a list of model variables. Let $\mathbf{f} \equiv\left[f_{p}\right]_{p \in \mathbf{P}}$ denote the vector of all PSR flows, such that $f_{p}$ denotes the flow on a specific parking search route $p \in \mathbf{P} \equiv\left[P_{r s}\right]$, where $P_{r s}$ is the PSR choice set for origin-destination pair $(r, s)$. Let $\tilde{c}_{p}(\mathbf{f})$ denote the perceived 
generalized costs of parking search route $p$. Then the PSR flow vector $\mathbf{f}$ is a PSR-SUE solution if it satisfies the following definition.

Definition 4. PSR-SUE problem. The parking search route flow vector $\mathbf{f}$ is a PSRSUE solution if and only if

$$
\begin{gathered}
f_{p}>\Delta F_{r s} \Rightarrow \tilde{c}_{p}=\tilde{c}_{r s}^{*}, \quad \forall p \in P, \\
f_{p}=0 \Rightarrow c_{p}(\mathbf{f})+\theta^{-1} \ln \Delta F_{r s} \geq \tilde{c}_{r s}^{*}, \quad \forall p \in P,
\end{gathered}
$$

s.t.

$$
\begin{gathered}
\Delta F_{r s} \geq 0, \quad \forall(r, s), \\
\sum_{p \in P_{r s}} f_{p}=F_{r s}, \quad \forall(r, s), \\
\left\{p \in P \mid f_{p}<0 \vee 0<f_{p}<\Delta F_{r s}\right\}=\emptyset,
\end{gathered}
$$

where

$$
\begin{gathered}
\tilde{c}_{r s}^{*}=\min _{p \in P_{r s}}\left\{\tilde{c}_{p}\right\}, \\
\tilde{c}_{p}=c_{p}(\mathbf{f})+\theta^{-1} \ln f_{p},
\end{gathered}
$$

such that $\tilde{c}_{p}$ is the stochastic equilibrium PSR cost, and $c_{p}(\mathbf{f})$ is the deterministic equilibrium PSR cost.

Conditions (1) and (2) formalize the PSR-SUE conditions given in Definition 3. Constraint (3) ensures relevant PSR flows above the threshold $\Delta F_{r s}$. This threshold, which is to be specified by the modeler, should be non-negative, and is often set to zero. Especially for the case of a SUE assignment, a positive value may be preferable for computational reasons, to avoid dealing with negligibly small route flows (see also the discussion in Watling et al. (2015)). Constraint (4) ensures that all travel demand for origin-destination pair $(r, s)$ is assigned to a parking search route. Constraint (5) is a generalization of the non-negative flow constraint, and here also excludes non-zero flows below the threshold. Equation (6) defines the minimum perceived generalized costs for all PSRs for an origin-destination pair. Equation (7) defines the perceived generalized costs for a specific PSR, where $\theta$ denotes the logit scale parameter, such that if $\theta \rightarrow \infty$ then parking search route choice is completely deterministic. Equation (7) entails that the PSR choices follow a logit choice model based on Random Utility Maximization (as shown by Bliemer et al. 2014).

The PSR-SUE formulation given by Equations (1)-(7) relates to the multinomial logit choice model that does not account for possible correlation in any factors apart from those specified in the generalized cost function. If necessary, the latter can be incorporated by adjusting Equations (2) and (7) accordingly.

\section{Generalized costs}

Next, without loss of generality, let $c_{p}(\mathbf{f})$ be composed of the expected utility associated with parking at one of the locations along the parking search route, and the expected utility associated with driving along the various route segments that constitute the parking search route. Let $V_{\pi}(\mathbf{f})$ denote the utility associated with parking at parking location $\pi$, and let $p(i)$ denote the $i^{\text {th }}$ parking location for PSR $p$. 
Let $V_{\delta}(\mathbf{f})$ denote the utility associated with driving along route segment $\delta$, and $(p(i), p(j))$ denote the route segment(s) connecting the $i^{\text {th }}$ and $j^{\text {th }}$ parking locations for PSR $p$. The generalized cost for PSR $p$ is then determined as,

$$
\begin{gathered}
c_{p}(\mathbf{f})=\sum_{i=1}^{i=I_{p}} \prod_{j=0}^{j=i-1}\left[1-\psi_{p(j)}\right] \cdot\left[-V_{p(i-1), p(i)}(\mathbf{f})-V_{p(i)}(\mathbf{f}) \cdot \psi_{p(i)}\right]+ \\
\prod_{i=1}^{i=I_{p}}\left[1-\psi_{p(i)}\right] \cdot \Lambda,
\end{gathered}
$$

where $I_{p}$ denotes the total number of parking locations included in $p$, and where $\psi_{\pi}$ denotes the probability of finding a vacant parking spot at location $\pi$, with $\psi_{p(0)}$ defined as 0 , and where $p(0)$ is defined as the origin $r$ of the PSR such that $V_{p(0), p(1)}$ denotes the utility of the route segment from the origin to the first parking location. Equation (8) thus consists of the sum of two terms. The first term describes, summed for all subsequent parking locations, the probability of arriving at this parking location (which equals the probability of not finding a vacant spot at any of the previous parking locations, and is thus given by the product of the complements of the parking probabilities at the previous locations) multiplied with the negative of the expected utility of driving to that parking location, and with the negative of the expected utility of parking multiplied with the probability of parking at that location. The second term in Equation (8) captures the costs of not finding a vacant parking spot at any of the considered parking locations, and is thus given by the product of the complements of the parking probabilities for all locations in $p$ multiplied with the associated costs hereof denoted by $\Lambda$ (which for modeling convenience can be set to any high value). Hence, the second term captures drivers' tendency towards a PSR that overall gives a sufficiently high probability of successfully finding a parking spot.

For sake of parsimony, and considering that we are predominantly interested in parking characteristics, in this paper we assume the utility associated with a specific route segment to be determined as,

$$
V_{\delta}(\mathbf{f})=\beta_{t} \cdot \tau_{\delta},
$$

where $\tau_{\delta}$ denotes the travel time of the route segment, and $\beta_{t}$ is the travel time parameter. Travel times can be computed using any network loading model, e.g., a microsimulation traffic model as used in the model application in this paper (with as input the traffic flow vector consistent with the PSR-SUE problem defined by Equations (1)-(7)). Equation (9) can easily be extended to include other utility attributes, such as travel costs and travel time reliability.

The utility associated with a specific parking location has been derived in an earlier study by the authors (Chaniotakis and Pel, 2015) and is determined as,

$$
V_{\pi}(\mathbf{f})=\beta_{c} \cdot \gamma_{\pi}+\beta_{w} \cdot \omega_{\pi}+\beta_{m} \cdot \mu_{\pi}+\beta_{y^{0}} \cdot \psi_{\pi}^{0}+\beta_{y^{\sigma}} \cdot \psi_{\pi}^{\sigma},
$$

where $\gamma_{\pi}, \omega_{\pi}, \mu_{\pi}, \psi_{\pi}^{0}$ and $\psi_{\pi}^{\sigma}$ denote respectively: the parking fee, walking distance from parking location to destination $s$, the parking type distinguishing on-street curbside parking and off-street parking (garages), the probability of parking availability upon arrival at the parking location, and the probability of parking availability within 
a maximum admissible search time denoted by $\sigma$. Parking fees, walking distances, and parking types are exogenous. Parking probabilities are endogenous as these depend on the chosen parking search routes. Parking probabilities are determined via a queuing model presented next. The parameters $\beta_{c}, \beta_{w}, \beta_{m}, \beta_{y^{0}}$ and $\beta_{y^{\sigma}}$ relate to their corresponding attributes. These parameters, together with $\beta_{t}$ in Equation (9) have been estimated in (Chaniotakis and Pel, 2015) using a stated choice experiment within the context of drivers' choosing a single parking location.

The parking probabilities in Equation (8) represent the probability of finding a vacant parking spot at that location. Hence, $\psi_{\pi}$ in Equation (8) is equivalent to $\psi_{\pi}^{\sigma}$ in Equation (10). Chaniotakis and Pel (2015) found that drivers are, on average, willing to spend 8 minutes searching for a parking spot before abandoning the location and continuing to a next parking location. This average search time is used here for sake of parsimony, however the findings by Chaniotakis and Pel (2015) suggest that search times might differ according to parking type. The latter can be incorporated by adjusting Equation (10) accordingly, such that $\sigma \rightarrow \sigma\left(\mu_{\pi}\right)$.

\section{Parking probabilities}

We propose a queuing model to determine the probability of finding a vacant parking spot after a certain amount of search time $\sigma$, where no search time (i.e., $\sigma=$ 0 ) yields the probability upon arrival. The appropriate queuing model is characterized by: the arrival process, the service mechanism, and the queue discipline.

The arrival process is endogenous. Let $a_{\pi}\langle k, k+\kappa\rangle$ denote the expected number of drivers that arrive at parking location $\pi$ during the period $\langle k, k+\kappa\rangle$. Then,

$$
\begin{aligned}
a_{\pi}\langle k, k+\kappa\rangle & =\sum_{p \in \mathbf{P}} f_{p} \cdot \sum_{i=1}^{i=I_{p}} \prod_{j=0}^{j=i-1}\left[1-\psi_{p(j)}^{\sigma}\right] \cdot \eta_{\pi, p(i), k}, \\
\text { where } \quad \eta_{\pi, p(i), k} & =\left\{\begin{array}{cc}
1 & \text { if } \pi=p(i) \wedge \tau_{o, p(i)}+(i-1) \cdot \sigma \in\langle k, k+\kappa\rangle \\
0 & \text { otherwise }
\end{array} .\right.
\end{aligned}
$$

That is, the expected number of arrivals at a parking location is given by the sum of all PSR flows multiplied with their respective probabilities that drivers arrive at that parking location within that time interval, where the latter probabilities are given by the probability of not finding a vacant spot at any of the previous parking locations multiplied with $\eta_{\pi, p(i), k}$ which is 1 only when the time interval of interest includes the arrival time of drivers' following PSR $p$, where the latter is given by the route travel time towards location $\pi$ (denoted by $\tau_{o, p(i)}$ ) plus the sum of the search times $\sigma$ spent at previously visited parking locations (given by $(i-1) \cdot \sigma$ ).

Note that departure times are omitted for sake of clarity in the notation (i.e., the current notation is consistent when all drivers depart at $k=0$ ). If necessary, departure times can be incorporated into the model formulation by the following adjustments: define $p$ as departure time specific, ensure in Equation (11) that in the condition for $\eta_{\pi, p(i), k}=1$ the departure time is added to the travel and search time, and define in Equations (1)-(7) the PSR set $P_{r s}$ and the demand $F_{r s}$ both as departure time specific.

The service mechanism of a specific parking location is exogenous. It entails the 
number of available servers and the service times (distribution). The former equals the number of parking spots at that location, while the latter equals the (distribution of) parking durations.

The queue discipline at a specific parking location is exogenous. It entails the queue priority and queuing behavior. The queue priority may either follow a firstcome first-served rule (which seems appropriate for off-street parking) or a random process (which seems appropriate for on-street parking). The queuing behavior here includes reneging where drivers will decide to leave the queue after the maximum admissible search time has passed.

The resulting parking queuing model can be classified using Kendall notation as G/G/c/\{FCFS, SIRO\}, where c equals the parking capacity at the parking location. This type of queuing model has no closed-form expression for the probability of being served (i.e., the complement of the probability of reneging) and hence needs to be approximated numerically (see Barrer, 1957 for first-come first-served (FCFS) priority; see Movaghar, 1998 for service in random order (SIRO) priority). Here we approximate the parking probabilities for any given parking location through simulation. Note that by computing the parking probabilities via simulating a queuing model, we can solve for any type of arrival and departure process, which is needed because these processes will most likely not adhere to any statistical distribution function, as one parking location takes demand from several PSR such that its arrival rate is the sum of the departure rates of preceding parking locations.

Considering a parking location, the parking probability at any specific arrival time and for any specific search time can be computed using the cumulative curves of arrivals entering the system and departures exiting the system. These cumulative curves are output of the simulation run of the queuing model. More specifically, let $A(k)$ denote the cumulative arrivals at the tail of the queue until time $k$, and let $D(k)$ denote the cumulative departures both from the queue due to reneging and from the servers (after being served) until time $k$. For the following, consider Fig. 2. 


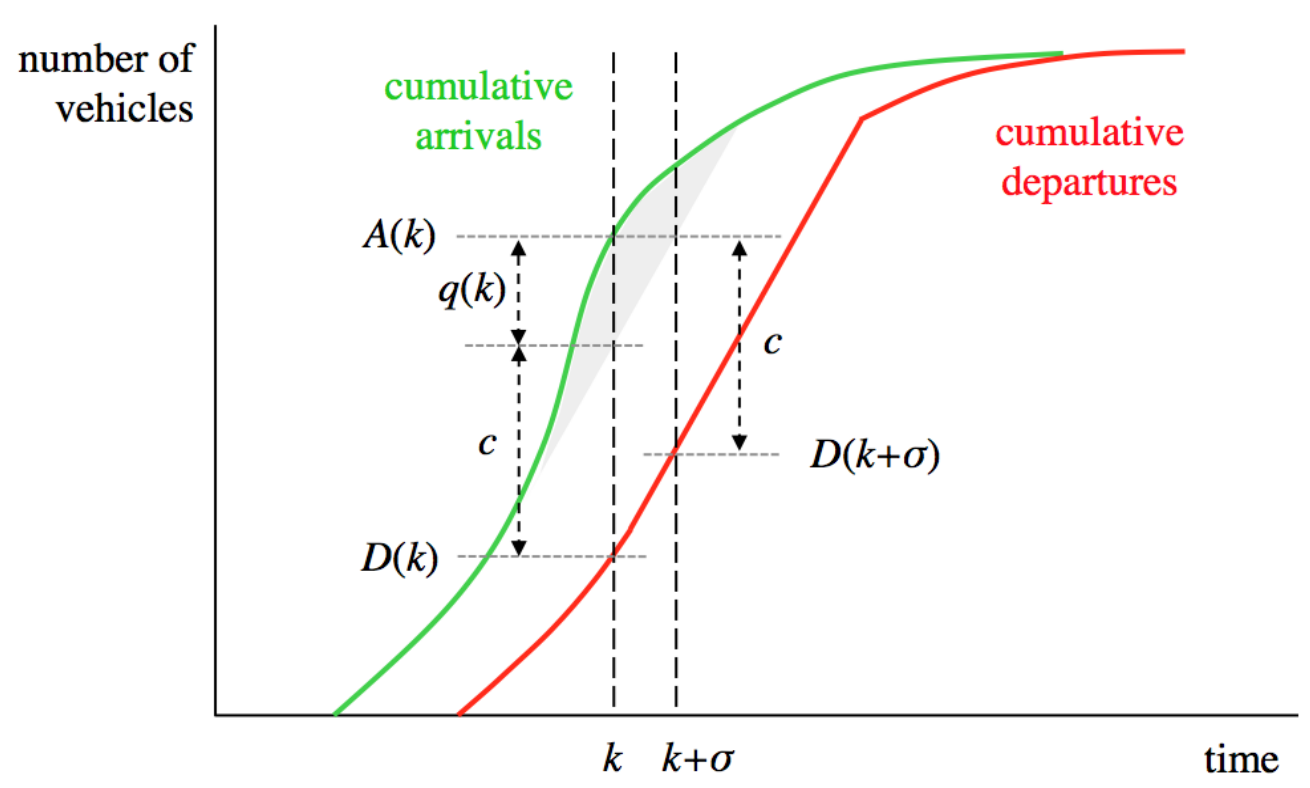

Fig. 2. Illustration of computation of expected search time using cumulative curves of arrivals and departures.

Under FCFS priority, we can look at the 'priority queue' that a (virtual) driver $d$ would encounter when arriving at time $k$, which equals $q(k)=A(k)-c-D(k)$, where $\mathrm{c}$ is the number of servers (i.e., the parking capacity). This priority queue represents the drivers who have priority over driver $d$ regarding being served (as we are considering FCFS). After time $\sigma$, this queue will have dissolved by an amount of $D(k+\sigma)-D(k)$. Thus parking probabilities under FCFS are computed as,

$$
\psi^{\sigma}(k)=\operatorname{Pr}(A(k)-c \geq D(k+\sigma)) .
$$

Note that this holds under the condition that any departure during $\langle k, k+\sigma\rangle$ is a departure from the priority queue, and not a departure from the complementary queue that may have accumulated after the arrival of driver $d$. It is easy to see that this always holds, since no driver in the complementary queue can have passed their maximum search time (as $k^{*}+\sigma>k+\sigma, \forall k^{*}>k$ ), nor can have been served (as there is FCFS priority).

Under SIRO priority, we need to look at the probability that a (virtual) driver $d$ arriving at time $k$ would at any time during $\langle k, k+\sigma\rangle$ be randomly selected to be served. To this end, let $S(k)$ denote the cumulative curve of the number of drivers that have been or are being served until time $k$ (which is also output of the simulation run of the queuing model). During any small interval $\langle x, x+d x\rangle$, the additional number of drivers that can be served is $S(x+d x)-S(x)$, while the drivers waiting to be served is $q(x)=\max \{0, A(x)-c-D(x)\}$. Hence the probability of driver $d$ being selected is $\min \{1,[S(x+d x)-S(x)] / q(x)\}$. Thus parking probabilities under SIRO are computed as,

$$
\psi^{\sigma}(k)=1-\int_{k}^{k+\sigma} \ln [1-\xi] d x
$$




$$
\text { where } \xi=\min \left\{1, \frac{S(x+d x)-S(x)}{\max \{0, A(x)-c-D(x)\}}\right\} \text {. }
$$

In case of FCFS priority and a deterministic arrival and departure process, then the parking probabilities are binary. When these processes are stochastic, or in case of SIRO priority, then the parking probabilities are true probabilities. For the stochastic case, these probabilities can be constructed using multiple simulation runs in a Monte Carlo fashion. This is shown in the following example.

To demonstrate the parking queuing model, Fig. 3 shows an example of the timevarying parking probability as a function of the maximum admissible search time. These probabilities are derived by simulating the queuing model for the conditions at a specific parking location. For this example we considered a 10-hour period with the following conditions:

- (Arrival process.) The arrival process is Poisson distributed with hourly rates given as $90,110,110,140,120,110,90,50,20,10$ vehicles per hour.

- (Service mechanism.) The number of servers (i.e. the parking capacity) is 250 . The parking duration is Exponential distributed with mean 150 minutes (such that the departure process is Poisson distributed).

- (Queuing discipline.) The queue priority is FCFS. The queue behavior includes reneging when the maximum search time has passed, where the model is run for various search times up to 10 minutes, $\sigma \in[0,10]$.

Note that in this example the arrival process and service mechanism both include a stochastic component. We therefore run 3000 simulations (of the full 10 hour period) with different random seeds and compute the expected parking probabilities shown in Fig. 3 by averaging the parking probabilities computed in the individual simulation runs using Equation (12).

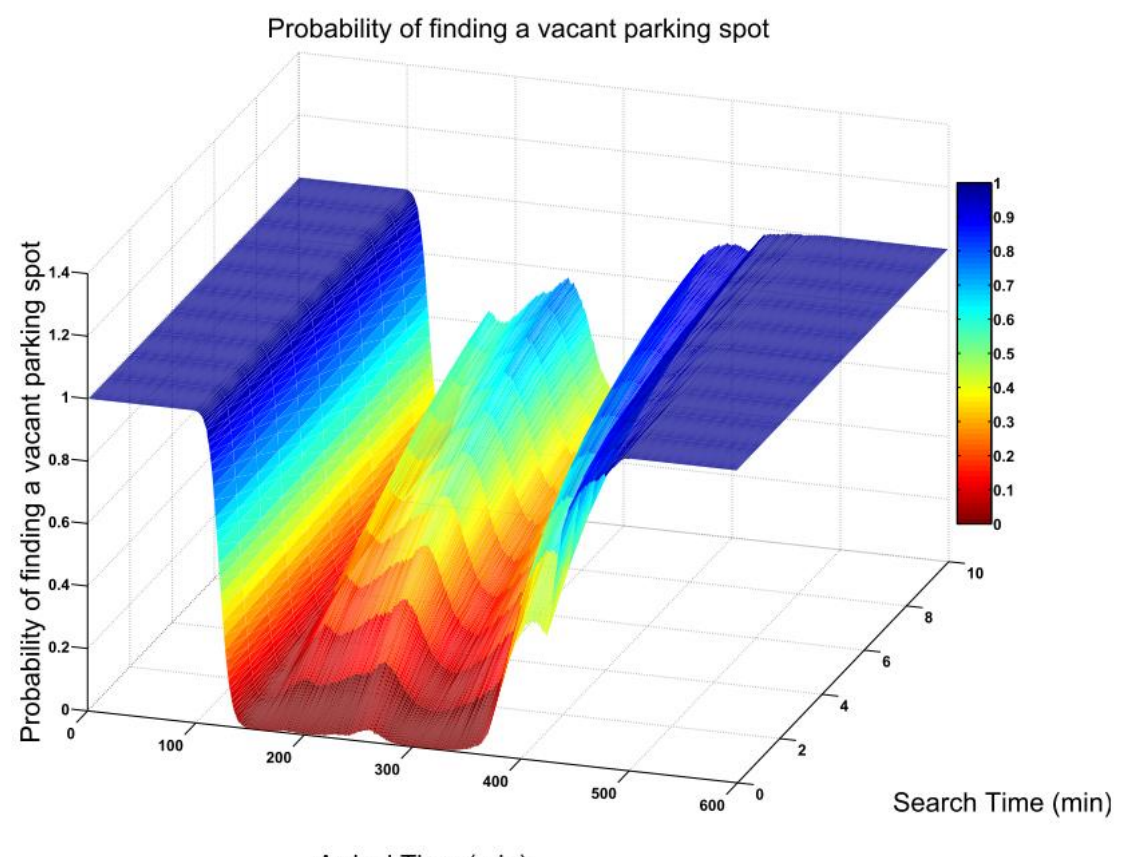

Arrival Time (min) 
Fig. 3. Example of the time-varying parking probability as a function of the maximum admissible search time. Parking probabilities are derived from 3000 simulation runs of the parking queuing model.

\section{Note 1: Non-atomic model formulation}

All variables in the proposed model are macroscopic, in terms of flows (i.e., PSR flows, traffic flows, arrival flows, queuing flows) and thus the model formulation is non-atomic. The queuing model can be solved as either atomic or non-atomic. The search process can relate to the behavior of either an atomic driver/vehicle or a nonatomic traffic flow.

The PSR-SUE model is in principle non-atomic, just like most route choice models, and can be combined either with a non-atomic macroscopic traffic flow model or with an atomic microscopic traffic simulation model. In the experiments in Section 3.1 we apply the PSR-SUE model while using a (non-atomic) macroscopic traffic flow model. Here we compute float-value flows and choice probabilities. In the application in Section 3.2 we apply the PSR-SUE model while using a (atomic) microscopic traffic simulation model. Here we compute integer-value flows and choice probabilities.

\section{Note 2: Time-dependent travel demand and traffic flows}

As mentioned earlier, we have omitted any time index in the model formulation for sake of clarity, apart from the computation of the parking probabilities in Equation (12) and (13) where $k$ is the arrival time at a parking location. If needed, one could include time-dependent travel demands and compute time-dependent traffic flows, travel times, etc. in the same way as done in a traditional dynamic traffic assignment model. Typically, the time-dependent travel demand is exogenous and fixed, while the time-dependent route flows are endogenous and in equilibrium. That is, drivers choose their optimal (parking search) route in response to the time-dependent traffic conditions, but their departure time is insensitive to these traffic conditions.

Furthermore, similar to a traditional dynamic traffic assignment model where drivers choose their route based on the (day-to-day average) time-dependent route travel times as would be experienced given their (fixed) departure time, here we assume drivers choose their parking search route based on the (day-to-day average) time-dependent route travel times and time-dependent probabilities of parking availability. The time-dependent (route) travel times are computed by the dynamic traffic flow model. The time-dependent probabilities of parking availability are computed by the previous queueing models, where the time-dependent arrival process is the result of the time-dependent travel times and (unsuccessful) search times at earlier parking locations along the PSR.

\section{Note 3: Inclusion of miscellaneous traffic flows}

The model proposed in this section solves the network assignment problem for the (parking search route) flow vector $\mathbf{f}$. Traffic flows other than searching for parking - 
e.g. through-traffic or traffic departing from the parking locations - can however be included straightforwardly. Any travel demand with a given origin and destination for which (searching for) parking is not relevant, can simply be added to flow vector $\mathbf{f}$. For this travel demand, the 'parking search route' simplifies to a simple route between origin and destination (as according to Corollary 1 below, given that the destination absorbs this traffic flow with probability equal to 1), and correspondingly the assignment of this travel demand simplifies to the traditional (SUE) route assignment problem. By including these miscellaneous traffic flows into flow vector $\mathbf{f}$, these traffic flows are jointly simulated in the traffic flow model, and hence we also inherently account for any interactions between this miscellaneous traffic and the parking search traffic (i.e. travel time delays induced by cruising traffic) while solving the equilibrium network assignment problem.

\subsection{PSR-SUE solution method}

To solve the PSR-SUE problem we propose an algorithm based on the PSR-SUE definition and conditions, given by Definitions $(2,3)$. The basic notion of the algorithm is to iteratively reassign traffic flows on any used PSR towards any PSR with lower perceived generalized costs. Across iterations, the convergence towards stochastic user equilibrium is checked via a gap function based on the PSR-SUE problem, given by Definition (4). The following relative gap function, adapted from Bliemer et al. (2014), will reach zero upon convergence towards an SUE:

$$
\begin{gathered}
\varepsilon=\frac{\sum_{(r, s)} \sum_{p \in P_{r s}} f_{p}\left(\tilde{c}_{p}-\phi_{r s}\right)}{\sum_{(r, s)} F_{r s} \phi_{r s}}, \\
\text { where } \phi_{r s}=\min _{p \in P_{r s}}\left\{c_{p}(\mathbf{f})+\theta^{-1} \ln \left[\max \left\{\Delta F_{r s}, f_{p}\right\}\right]\right\},
\end{gathered}
$$

where $\tilde{c}_{p}$ is the perceived generalized costs, which under the earlier assumption of logit based PSR choice behavior is defined as $\tilde{c}_{p}=c_{p}(\mathbf{f})+\theta^{-1} \ln f_{p}$, and where $\phi_{r s}$ denotes the minimum perceived generalized costs against which the gap function is defined. Recall that the PSR-SUE assignment includes the constraint on any non-zero flow being no less than threshold $\Delta F_{r s}$. Therefore, $\phi_{r s}$ is the minimum of the PSR costs on any used route or the PSR costs that would be experienced by a flow of $\Delta F_{r s}$ on any currently unused route. (Note that the latter check is omitted from the original definition in (Bliemer et al., 2014), where instead they use a threshold-dependent route set $P_{r s}^{\Delta} \subseteq P_{r s}$ from which they exclude any route with $f_{p}=0 \Rightarrow \tilde{c}_{p} \leq \min _{p \in P_{r s}}\left\{\tilde{c}_{p}\right\}$ and $f_{p}=\Delta F_{r s} \Rightarrow \tilde{c}_{p}>\min _{p \in P_{r s}}\left\{\tilde{c}_{p}\right\}$. However, that approach leads to both appending and pruning the route choice set while solving the assignment problem. This can be computationally disadvantageous and is not needed with our calculation of $\phi_{r s}$.)

The proposed algorithm contains three sub-problems, namely the logit choice model to determine PSR flows, the network loading model to determine travel times, and the parking queuing model to determine parking probabilities. Note the following interdependencies between the PSR flow vector $\mathbf{f} \equiv\left[f_{p}\right]$, the travel time vector $\boldsymbol{\tau} \equiv$ $\left[\tau_{\delta}\right]$, and the parking probability vector $\boldsymbol{\Psi} \equiv\left[\psi_{\pi}\right]$ : 
- (Logit choice model.) The PSR flows depend on the PSR costs, which are a function of the travel times and the parking probabilities, computed using Equations (1)-(2), (7)-(10). Thus, $\mathbf{f}=\mathbf{f}(\boldsymbol{\tau}, \boldsymbol{\Psi})$.

- (Network loading model.) The travel times depend on the traffic flows, which are a function of the PSR flows and the parking probabilities, where the probability for a flow to traverse a route segment $(p(i), p(j))$ is computed as $\prod_{x=0}^{x=i} 1-\psi_{p(x)}$. Thus, $\boldsymbol{\tau}=\boldsymbol{\tau}(\mathbf{f}, \boldsymbol{\Psi})$.

- (Parking queuing model.) The parking probabilities depend on the arrival processes at the parking locations, which are a function of the PSR flows and the travel times, computed using Equation (11). Thus, $\boldsymbol{\Psi}=\boldsymbol{\Psi}(\mathbf{f}, \boldsymbol{\tau})$.

We propose to resolve these interdependencies in any intermediate iteration $\alpha$ in a forward updating fashion by assuming $\mathbf{f}^{\alpha}=\mathbf{f}^{\alpha}\left(\tau^{\alpha-1}, \boldsymbol{\Psi}^{\alpha-1}\right), \tau^{\alpha}=\tau^{\alpha}\left(\mathbf{f}^{\alpha}, \boldsymbol{\Psi}^{\alpha-1}\right)$, and $\boldsymbol{\psi}^{\alpha}=\boldsymbol{\psi}^{\alpha}\left(\mathbf{f}^{\alpha}, \boldsymbol{\tau}^{\alpha}\right)$. This way consistency is guaranteed upon convergence, due to the fact that then $\tau^{\alpha}=\tau^{\alpha-1}$ and $\psi^{\alpha}=\psi^{\alpha-1}$.

We propose the following solution algorithm for the PSR-SUE problem, which uses as input an explicit PSR choice set $\mathbf{P} \equiv\left[P_{r s}\right]$. The way in which we propose to generate this PSR choice set and possibly extend it during Step 6 is discussed hereafter.

\section{Algorithm 1. PSR-SUE solution algorithm.}

Input: PSR choice sets $\mathbf{P} \equiv\left[P_{r s}\right]$, travel demand $\mathbf{F} \equiv\left[F_{r s}\right]$, network characteristics, parking characteristics, and choice parameters $\{\boldsymbol{\beta}, \theta, \sigma\}$.

Step 0: Initialize. Assume initial (null) flow assignment $\mathbf{f}^{0}=0$. Assume initial (freeflow) travel times $\boldsymbol{\tau}^{\mathbf{0}}=\boldsymbol{\tau}(\mathbf{f}=0)$. Assume initial (sufficient) parking probabilities $\boldsymbol{\Psi}^{0}=1$. Set $\alpha:=1$.

Step 1: Compute logit choice model. Compute the intermediate PSR flows $\tilde{\mathbf{f}}^{\alpha}$ using the multinomial logit model using the PSR costs given by Equations (7)-(10), with travel times given by $\boldsymbol{\tau}^{\alpha-1}$ and parking probabilities given by $\boldsymbol{\Psi}^{\alpha-1}$.

Step 2: Update PSR flows. Compute the new averaged PSR flows as $\mathbf{f}^{\alpha}=\mathbf{f}^{\alpha-1}+$ $\alpha^{-\lambda}\left[\tilde{\mathbf{f}}^{\alpha}-\mathbf{f}^{\alpha-1}\right]$, for a given $\lambda \in[0,1]$.

Step 3: Compute network loading model. Compute the travel times $\boldsymbol{\tau}^{\alpha}$ using any network loading model, with travel demand given by $\mathbf{f}^{\alpha}$ and probabilities of being absorbed by parking locations given by $\boldsymbol{\Psi}^{\alpha-1}$.

Step 4: Compute parking queuing model. Simulate the queuing model G/G/c/\{FCFS, SIRO $\}$, with the arrival processes given by Equation (11), the travel demand given by $\mathbf{f}^{\alpha}$ and the travel times given by $\boldsymbol{\tau}^{\alpha}$. Compute the parking probabilities $\boldsymbol{\Psi}^{\alpha}$ using Equation (12) or (13).

Step 5: Update PSR costs. Compute the new PSR costs given by Equations (7)-(10), with travel times given by $\boldsymbol{\tau}^{\alpha}$ and parking probabilities given by $\boldsymbol{\Psi}^{\alpha}$.

Step 6: (optional) Update PSR choice set. If necessary, for each origin-destination 
pair, append the choice set with any PSR $q$ whose stochastic equilibrium cost is not larger than the current minimum perceived generalized costs within the PSR choice set, i.e. if $c_{q}\left(\mathbf{f}^{\alpha}\right)+\theta^{-1} \ln \Delta F_{r s} \leq \min _{p \in P_{r s}}\left\{\tilde{c}_{p}\right\}$.

Step 7: Check convergence. Compute the relative gap $\varepsilon^{\alpha}$ using Equation (14). If $\varepsilon^{\alpha}$ is smaller than a pre-determined threshold, then stop. Otherwise, set $\alpha:=$ $\alpha+1$ and return to Step 1 .

Note that in Step 2, $\lambda=1$ for traditional MSA, while $\lambda<1$ results in larger step sizes and may lead to accelerated convergence (see e.g., Taale and Pel, 2015).

\section{PSR choice set generation}

The proposed solution algorithm uses as input an explicit PSR choice set which thus needs to be pre-generated, and possibly extended during the assignment, as we will discuss later. Recall that a PSR according to Definition 1 is constructed of a sequence of route segments between pairs of consecutive parking locations. Alternative PSRs therefore will differ with respect to the parking locations included, the order in which these are included, and the route segments used between parking locations. We propose to exploit this understanding to generate a PSR choice set in a tractable stepwise procedure.

We can also exploit a feature of parking search routes that follows from Definition 1 and Equations (7)-(10), namely:

Proposition 1. For sufficiently large $\Lambda$, any parking search routes $p$ and $p^{*}$ for which holds that $(p(i-1), p(i))=\left(p^{*}(i-1), p^{*}(i)\right), \forall i=1, \ldots, I_{p}$ will have costs such that $c_{p}(\boldsymbol{f}) \geq c_{p^{*}}(\boldsymbol{f})$. Therefore appending a parking search route with additional route segments will not increase its PSR cost.

Proof. Given that $p$ is a subset of $p^{*}$, we can rewrite $p^{*} \equiv\left\{p,\left(p^{*}\left(I_{p}\right), p^{*}\left(I_{p^{*}}\right)\right)\right\}$. Or, in words, PSR $p^{*}$ is the set of $p$ and the route segments in $p^{*}$ between the last parking location in $p$, which is $p^{*}\left(I_{p}\right)$, and the last parking location in $p^{*}$, which is $p^{*}\left(I_{p^{*}}\right)$. Together with Equation (5) this gives,

$$
\begin{array}{r}
c_{p}(\mathbf{f})=\Upsilon_{1}^{I_{p}}+\Phi_{1}^{I_{p}} \cdot \Lambda, \quad \text { and } \\
c_{p^{*}}(\mathbf{f})=\Upsilon_{1}^{I_{p}}+\Upsilon_{I_{p}+1}^{I_{p^{*}}}+\Phi_{1}^{I_{p}} \cdot \Phi_{I_{p}+1}^{I_{p^{*}}} \cdot \Lambda .
\end{array}
$$

Therefor, $c_{p}(\boldsymbol{f}) \geq c_{p^{*}}(\boldsymbol{f})$ if,

$$
\Phi_{1}^{I_{p}}\left[1-\Phi_{I_{p}+1}^{I_{p^{*}}}\right] \Lambda \geq \Upsilon_{I_{p}+1}^{I^{*}}
$$

where $\quad \Upsilon_{x}^{y}=\sum_{i=x}^{i=y} \prod_{j=0}^{j=i-1}\left[1-\psi_{p(j)}\right] \cdot\left[-V_{p(i-1), p(i)}(\mathbf{f})-V_{p(i)}(\mathbf{f}) \cdot \psi_{p(i)}\right]$,

and $\Phi_{x}^{y}=\prod_{i=x}^{i=y}\left[1-\psi_{p(i)}\right]$.

Note that the term $\Phi_{1}^{I_{p}}\left[1-\Phi_{I_{p}+1}^{I_{p^{*}}}\right] \in[0,1]$, however, this term can only by zero when 
$\Phi_{1}^{I_{p}}=0$ or $\Phi_{I_{p}+1}^{I_{p^{*}}}=1$. Note that $\Phi_{1}^{I_{p}}=0$ if and only if there exists a $\psi_{p(i)}=1$ with $i \in\left[1, I_{p}\right]$, and therefore $\Upsilon_{I_{p}+1}^{I_{p^{*}}}=0$. Note that $\Phi_{I_{p}+1}^{I_{p^{*}}}=1$ if and only if $\psi_{p(i)}=0$ for all $i \in\left[I_{p+1}, I_{p^{*}}\right]$, and therefore $\Upsilon_{I_{p}+1}^{I_{p^{*}}}=0$. Hence, we have that if $\Phi_{1}^{I_{p}}\left[1-\Phi_{I_{p}+1}^{I_{p^{*}}}\right]=$ 0 , then $\Upsilon_{I_{p}+1}^{I_{p^{*}}}=0$, and if $\Phi_{1}^{I_{p}}\left[1-\Phi_{I_{p}+1}^{I_{p^{*}}}\right] \in\langle 0,1]$, then $\Upsilon_{I_{p}+1}^{I_{p^{*}}}$ is typically negative or small. Therefore when $\Lambda$ is sufficiently large, any increase in costs due to visiting additional parking locations will be lower than the decrease in costs due to a lower probability (i.e., penalty) of not finding a parking spot at all. This concludes the proof.

Proposition 1 states that there is no need to generate any PSR $p$ that is a subset of another PSR already included in the choice set, i.e. $p \subseteq p^{*} \in \mathbf{P}$.

The proof of Proposition 1 also shows the following property of a PSR:

Corollary 1. Any parking search routes $p$ and $p^{*}$ for which holds that ( $p(i-$ $1), p(i))=\left(p^{*}(i-1), p^{*}(i)\right), \forall i=1, \ldots, I_{p}$ and where $p$ includes a parking location for which $\psi_{p(i)}=1$, are equivalent. Therefore appending a parking search route with additional route segments beyond a parking location with parking probability equal to 1 is irrelevant.

Proof. If $\psi_{p(i)}=1$ for any $i \in\left[1, I_{p}\right]$, then $\Phi_{1}^{i}=\prod_{x=0}^{x=i}\left[1-\psi_{p(x)}\right]=0$. Therefore, as shown by the Proof of Proposition $1 \Upsilon_{I_{p}+1}^{I_{p^{*}}}=0$, due to the fact that the parking search route will be certainly aborted at parking location $p(i)$ and thus any following route segments will never be used. This concludes the proof.

We propose the following PSR choice set generation algorithm.

\section{Algorithm 2. PSR choice set generation algorithm.}

Step 1: Generate parking location set. Select the set of parking locations $\{\pi\}_{s}$ that do not exceed a non-compensatory level for any of the exogenous attributes in Equation (10) (e.g., locations within a maximum walking distance to the destination).

Step 2: Generate parking sequence set. For all pairs of parking locations $\left\{\pi^{*}, \pi\right\} \subseteq$ $\{\pi\}_{S}$ compute their shortest distance. Generate the $k$-shortest sequences $\{\boldsymbol{\pi}\}_{S}$, where each sequence is a permutation that contains all locations in $\{\pi\}_{S}$ (thus excluding incomplete sequences by Proposition 1), thereby prioritizing sequences that are efficiently ordered.

Step 3: Generate route segment sets. Generate the constrained $k$-shortest routes $\{\delta\}_{\left(\pi^{*}, \pi\right)}$ between all consecutive pairs of parking locations $\left\{\pi^{*}, \pi\right\} \subseteq\{\boldsymbol{\pi}\}_{S}$, with bounded costs compared to the shortest route (see Fiorenzo-Catalano and Van der Zijpp, 2001). 
Step 4: Compose PSR master choice set. Generate the PSR master choice set $P_{r s}$ using the constrained $k$-shortest route method by concatenating alternative route segments from Step 3 for the parking sequences from Step 2.

Note that in Step 2 if $\left\|\{\pi\}_{s}\right\|$ is sufficiently small, then this entails full enumeration when $\left\|\{\pi\}_{s}\right\| ! \leq k$. Note that in Step 3 although in most cases full enumeration will not be feasible, these route segment sets can be relatively rich given that we are generating routes that are confined within a small part of the network between pairs of parking locations.

Algorithm 2 generates the PSR choice set for a specific origin-destination pair, and hence needs to be executed per origin-destination pair. Nevertheless it is still rather efficient, because earlier computations can be reused. The route segment sets generated in Step 3 are specific to a pair of consecutive parking locations, and thus can be used in any parking sequence. And the parking sequence set in Step 2 is specific to a parking location set, which in turn is specific to a destination, and thus can be used for any origin-destination pair with this destination. For an efficient implementation of the PSR choice set generation algorithm all of these sets can be computed and stored in a lazy fashion.

Watling et al. (2015) discuss several disadvantages of using a pre-generated master choice set. We would like to argue that these disadvantages are avoided in cases where the master choice set can be generated using a deterministic method (ensuring repeatability) based on exogenous variables (ensuring consistency). Nevertheless, the proposed PSR choice set generation method involves sampling, and therefore the pregenerated master choice set might need to be dynamically extended during the iterative assignment procedure. This is done in Step 6 in Algorithm 1, where basically Step 4 of Algorithm 2 is repeated with updated costs.

\section{Model experiments and application}

In the following section we first run several experiments on synthetic networks that are specifically constructed to demonstrate the properties of the proposed PSR-SUE assignment model. Here, we focus on verifying the flows and costs of parking search routes, parking probabilities under equilibrium, and model convergence. Afterwards, we apply the model to a real-life setting of the Dutch city of Assen. In the model application we embed the PSR-SUE assignment model within a simulation framework to include aspects of background traffic, traffic signal control, rerouting behavior due to traffic information, and a myopic search process for on-street parking. We discuss some basic scenario analysis regarding parking search behavior.

\subsection{Experiments}

\section{Experiment I}

Consider the network shown in Fig. 4 with 1 origin-destination pair and 2 parking locations. The travel demand is 400 vehicles. Both parking locations share the 
following characteristics: 400 meters walking distance to destination, off-street parking, and a parking capacity of 200. The parking locations differ only according to parking fees, where $\mathrm{P} 1$ is $€ 2.30$ and $\mathrm{P} 2$ is $€ 3.00$. For simplicity, the travel time is calculated using the standard BPR-function, such that the travel time from the origin to either of the parking locations is approx. 15 minutes, and between the parking locations is approx. 5 minutes.

Solving the PSR-SUE for Experiment I yields the results shown in Table 1. For a relative gap of $\varepsilon \leq 1$ e- 3 the assignment converges within 10 iterations. As expected, PSR (P1, P2) has a higher flow than PSR (P2, P1), because P1 is preferred over P2 as it has lower parking cost. Due to this flow assignment, the parking probability at $\mathrm{P} 1$ drops below 1. Under equilibrated parking search routes, the probability of finding a parking spot at $\mathrm{P} 1$ is about $0.73-0.89$. That is, 226-273 vehicles follow PSR (P1, P2) while P1 has 200 capacity. When parking search route choice is more deterministic (i.e., the logit scale parameter is set to a larger value), then more drivers choose PSR $(\mathrm{P} 1, \mathrm{P} 2)$ as it has lower (deterministic) costs, and therefore the parking probability at $\mathrm{P} 1$ is lower in this case.

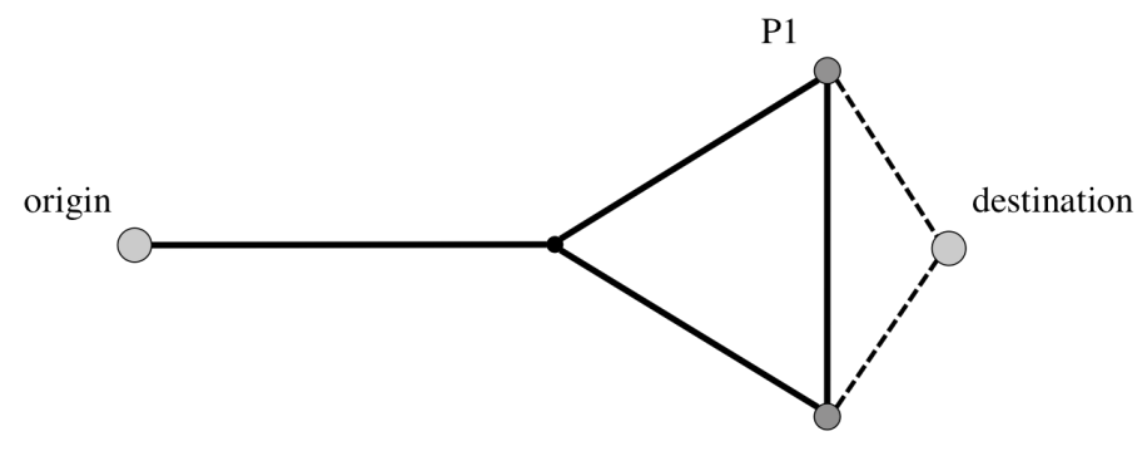

P2

Fig. 4. Synthetic network with 1 origin-destination pair and 2 parking locations, indicated as $\mathrm{P} 1$ and $\mathrm{P} 2$.

Table 1. PSR-SUE model results for Experiment I.

Logit scale parameter $(\theta)=1$

\begin{tabular}{ccccc}
\hline PSR $(p)$ & $\begin{array}{l}\text { deterministic equil. } \\
\text { PSR cost }\left(\tilde{c}_{p}^{*}(\mathbf{f})\right)\end{array}$ & $\begin{array}{l}\text { stochastic equil. } \\
\text { PSR } \operatorname{cost}\left(c_{p}(\mathbf{f})\right)\end{array}$ & $\begin{array}{l}\text { equilibrium } \\
\text { flow }\left(f_{p}\right)\end{array}$ & $\begin{array}{l}\text { relative gap } \\
(\varepsilon)\end{array}$ \\
\hline$(\mathrm{P} 1, \mathrm{P} 2)$ & 0.463 & 5.884 & 226.00 & $6.98 \mathrm{e}-4$ \\
$(\mathrm{P} 2, \mathrm{P} 1)$ & 0.734 & 5.893 & 174.00 & \\
\hline
\end{tabular}

Logit scale parameter $(\theta)=40$

\begin{tabular}{lllll}
\hline PSR $(p)$ & deterministic equil. & stochastic equil. & equilibrium & relative gap \\
& PSR cost $\left(c_{p}(\mathbf{f})\right)$ & PSR $\operatorname{cost}\left(c_{p}(\mathbf{f})\right)$ & flow $\left(f_{p}\right)$ & $(\varepsilon)$ \\
\hline
\end{tabular}




\begin{tabular}{llccc}
\hline$(\mathrm{P} 1, \mathrm{P} 2)$ & 0.714 & 0.855 & 273.00 & \\
$(\mathrm{P} 2, \mathrm{P} 1)$ & 0.734 & 0.855 & 127.00 & $1.71 \mathrm{e}-4$ \\
\hline \hline
\end{tabular}

\section{Experiment II}

Consider the network shown in Fig. 5 with 1 origin-destination pair and 3 parking locations. The travel demand is 400 vehicles. All parking locations are at 400 meters walking distance to the destination and provide off-street parking. The parking locations differ according to parking capacity and parking fee. These values are for P1: 150 capacity and $€ 3.00$ fee, for P2: 50 capacity and $€ 2.00$ fee, and for P3: 100 capacity and $€ 2.00$ fee. Once again the BPR-function is used for travel times, which are approx. 15 minutes from origin to $\mathrm{P} 1$ or $\mathrm{P} 2$, approx. 12 minutes from origin to $\mathrm{P} 3$, and approx. 4 minutes between parking locations.

Solving the PSR-SUE for Experiment II yields the results shown in Table 2. For a relative gap of $\varepsilon \leq 1$ e-3 the assignment converges within 15 iterations. As mentioned before, the endogenous parking probabilities depend on the arrival flows at the parking locations, which in turn depend both directly on the parking probabilities (at previous parking locations along the PSR) and on the PSR flows, while the PSR flows depend (through the choice model) on the parking probabilities. Interestingly, under equilibrated PSR-SUE conditions, the ranking among all PSRs follows the preference P3 (with intermediate parking probability $\psi_{3}=0.73$ and low fee $€ 2$ ), then P1 (with high parking probability $\psi_{1}=1.0$ and high fee $€ 3$ ), and lastly P2 (with low parking probability $\psi_{2}=0.46$ and low fee $€ 2$ ). Note in the arrivals at the parking locations that for any PSR where P2 or P3 is preceded by P1 that there is no arrival flow. This is due to the fact that the parking probability at $\mathrm{P} 1$ is $\psi_{1}=1.0$.

Similar as shown in Experiment I, setting the logit scale parameter $\theta$ to a higher value produces more deterministic PSR choice behavior and hence assigns more flows to those PSRs with lower deterministic costs. In this example, at P3 that would lead to more arrivals and hence a lower parking probability, while at P2 that would lead to less arrivals and hence a higher parking probability.

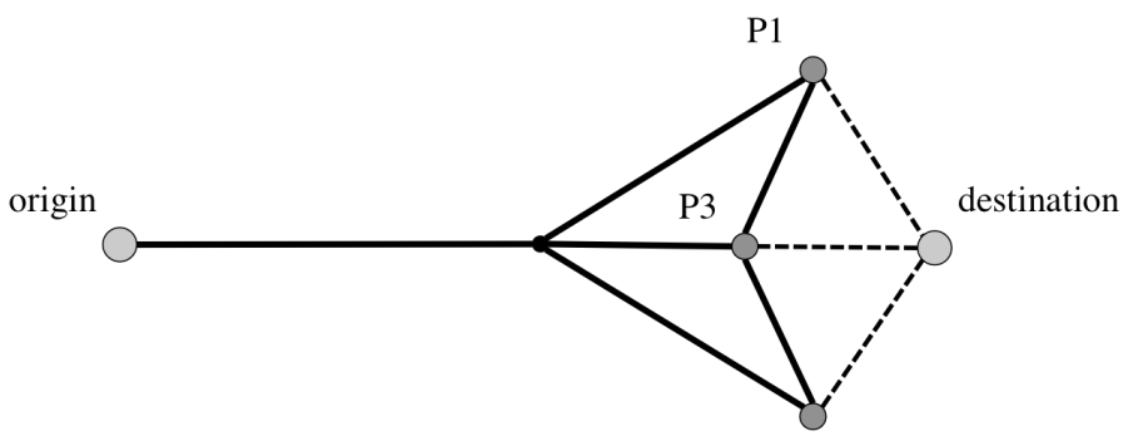

P2 
Fig. 5. Synthetic network with 1 origin-destination pair and 3 parking locations, indicated as $\mathrm{P} 1, \mathrm{P} 2$ and $\mathrm{P} 3$.

Table 2. PSR-SUE model results for Experiment II, for logit scale parameter $\theta=1$.

\begin{tabular}{lcccc}
\hline \hline PSR $(p)$ & $\begin{array}{l}\text { deterministic equil. } \\
\text { PSR cost }\left(\tilde{c}_{p}^{*}(\mathbf{f})\right)\end{array}$ & $\begin{array}{l}\text { stochastic equil. } \\
\text { PSR cost }\left(c_{p}(\mathbf{f})\right)\end{array}$ & $\begin{array}{l}\text { equilibrium } \\
\text { flow }\left(f_{p}\right)\end{array}$ & $\begin{array}{l}\text { relative gap } \\
(\varepsilon)\end{array}$ \\
\hline$(\mathrm{P} 1, \mathrm{P} 2, \mathrm{P} 3)$ & 0.404 & 4.270 & 47.74 & \\
$(\mathrm{P} 1, \mathrm{P} 3, \mathrm{P} 2)$ & 0.404 & 4.270 & 47.74 & \\
$(\mathrm{P} 2, \mathrm{P} 1, \mathrm{P} 3)$ & 0.478 & 4.270 & 44.34 & $3.27 \mathrm{e}-4$ \\
$(\mathrm{P} 2, \mathrm{P} 3, \mathrm{P} 1)$ & 0.370 & 4.270 & 49.38 & \\
$(\mathrm{P} 3, \mathrm{P} 1, \mathrm{P} 2)$ & 0.239 & 4.270 & 56.32 & \\
$(\mathrm{P} 3, \mathrm{P} 2, \mathrm{P} 1)$ & 0.272 & 4.270 & 54.48 & \\
\hline \hline
\end{tabular}

\begin{tabular}{lccc}
\hline \hline PSR $(p)$ & arrivals at P1 & arrivals at P2 & arrivals at P3 \\
\hline$(\mathrm{P} 1, \mathrm{P} 2, \mathrm{P} 3)$ & 47.74 & 0.00 & 0.00 \\
$(\mathrm{P} 1, \mathrm{P} 3, \mathrm{P} 2)$ & 47.74 & 0.00 & 0.00 \\
$(\mathrm{P} 2, \mathrm{P} 1, \mathrm{P} 3)$ & 44.34 & 0.00 \\
$(\mathrm{P} 2, \mathrm{P} 3, \mathrm{P} 1)$ & 23.92 & 49.38 & 26.64 \\
$(\mathrm{P} 3, \mathrm{P} 1, \mathrm{P} 2)$ & 7.25 & 0.00 & 56.32 \\
$(\mathrm{P} 3, \mathrm{P} 2, \mathrm{P} 1)$ & 15.34 & 14.83 & 54.48 \\
\hline total arrivals & 8.00 & 108.55 & 137.44 \\
\hline $\begin{array}{l}\text { parking } \\
\text { probability }(\psi)\end{array}$ & 150.00 & 0.46 & 0.73 \\
\hline \hline
\end{tabular}

\section{Experiment III}

For the third experiment, consider the Assen network shown in Fig. 6 where we select 7 origin-destination pairs and 6 parking locations. Here we look at the dynamic case and simulate the PSR-SUE flows for a time horizon of 90 minutes. The total travel demand is approx. 3400 vehicles and follows a 'peak period' profile gradually increasing from 0 to 3200 vehicles per hour within [0 $\mathrm{min}, 60 \mathrm{~min}$ ], and then gradually decreasing again back to 2400 vehicles per hour within [60 $\mathrm{min}, 90 \mathrm{~min}$ ]. Furthermore, we look at the situation in which the total parking capacity is 
insufficient, with a total capacity of 3256 . Note that in the previous experiments the total travel demand does not exceed the total parking capacity and therefore there will be at least one parking location with parking probability 1 . Then Proposition 2 states that all PSRs yield successful parking and thus the value of $\Lambda$ in Equation (5) is irrelevant. In Experiment III this is no longer the case.

Apart from parking location P22 which has free parking, all other locations have parking fees of $€ 1.70$. All parking locations are off-street parking. Walking distances to the destinations are measured on the map and are between 200 and 1400 meters. And parking capacities vary between 250 and 840 .

Travel times are calculated using a simple dynamic network loading model without consideration for spillback. Solving the PSR-SUE flow assignment for Experiment III requires generating about 120 PSRs. The number of iterations for convergence to an acceptable relative gap is approx. 80. This appears as a rather slow convergence, but this is solely due to the later time periods with high arrival flows and low parking probabilities at parking locations, when (some) PSRs do not guarantee finding a parking spot. For earlier time periods where parking probabilities do not deviate (much) from 1, the equilibrium PSR flows are found within a few iterations. Table 3 shows the dynamic parking flows, which seem plausible. Parking location P22, which offers free parking, fills up quickest. The other parking locations fill up at a similar pace, which is explainable from the fact that their characteristics do not differ much. Parking locations P10, P13 and P14 have below average capacity and are full before the end of the time horizon, where P13 has the lowest capacity and fills up slightly quicker than the other two locations. Parking locations P9 and P15 are slightly farther away from the city center and have the largest capacities, and hence fill up last. It is generally observed that once a parking location has a very low parking probability (or is full) then those PSRs with that parking location as one of their first locations to be visited are quickly abandoned due to high expected costs. 


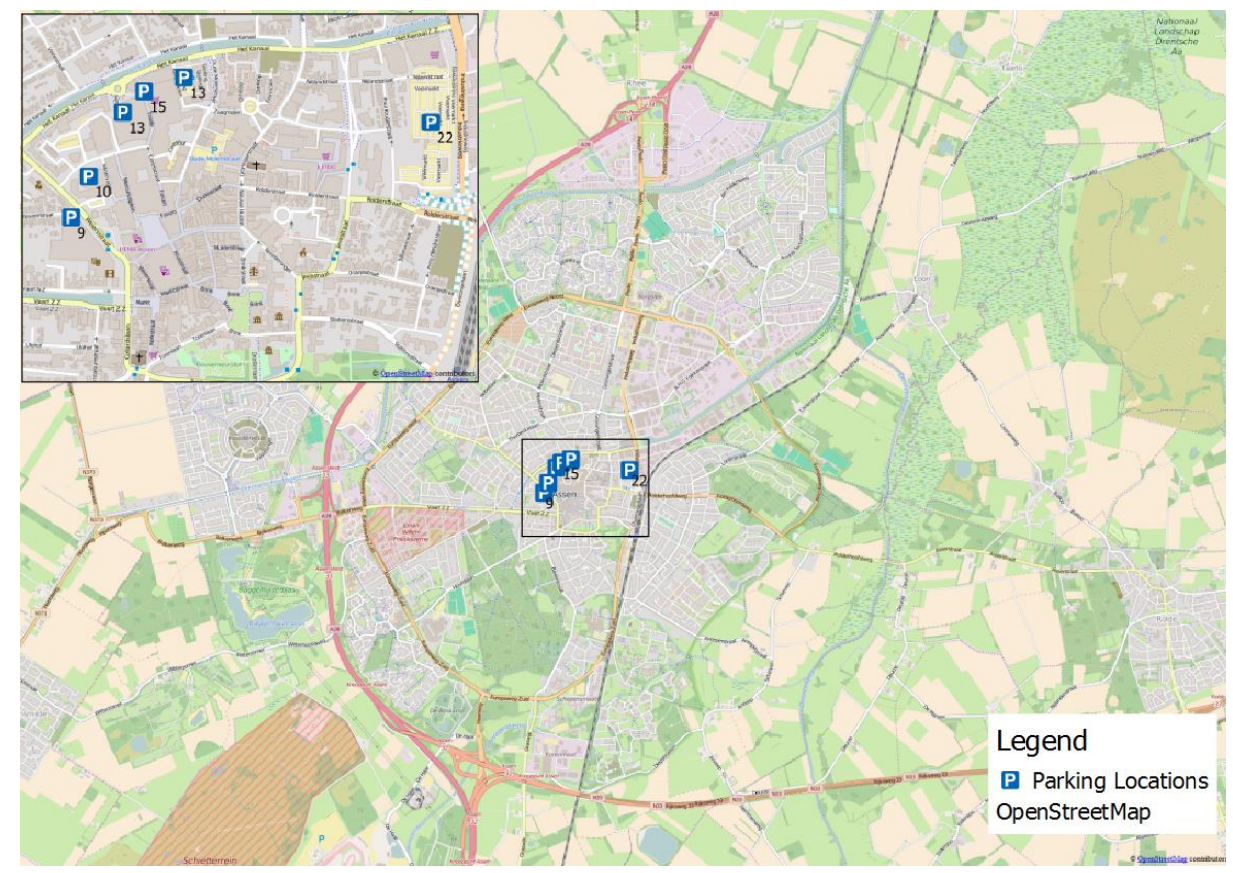

Fig. 6. Assen network with 6 parking locations, indicated as P9, P10, P13, P14, P15, and P22.

Table 3. PSR-SUE model results for Experiment III.

\begin{tabular}{ccccccc}
\hline \hline $\begin{array}{c}\text { time } \\
\text { period } \\
{[\mathrm{min}]}\end{array}$ & P9 & P10 & P13 & P14 & P15 & P22 \\
\cline { 2 - 7 } & 28 & 29 & 28 & 29 & 30 & 104 \\
$0-15$ & 41 & 42 & 43 & 43 & 42 & 161 \\
$15-30$ & 71 & 71 & 69 & 70 & 71 & 237 \\
$30-45$ & 143 & 151 & 111 & 143 & 145 & 98 \\
$45-60$ & 266 & 167 & 0 (full) & 79 & 279 & 0 (full) \\
$60-75$ & 191 & 0 (full) & 0 (full) & 0 (full) & 274 & 0 (full) \\
$75-90$ & & & & & & \\
\hline \hline
\end{tabular}

\subsection{Application}

To demonstrate the practical applicability of the proposed model, it is applied to the real-life setting of the city of Assen in the Netherlands. The network is shown in Fig. 6 (and includes another 5 parking locations in the vicinity of the city center). The case study considers 296 origin-destination pairs with travel demand, and a 2-hour demand matrix following a more or less triangular profile with in total about 4000 vehicles that intend to park somewhere in the vicinity of the city center, and in total about 11500 vehicles that have destinations throughout the city but do not make use 
of the parking locations (i.e. background traffic). The road network includes 11 parking destinations with capacity between 85 and 840 that in total provide a parking capacity of 3256. Parking fees vary between free parking and $€ 2.00$ per hour. We consider the case of parking for shopping and accordingly assume that parking durations follow a Uniform distribution between 30 and 90 minutes. We would like to emphasize that in this case study the traffic network and parking facilities including their characteristics do represent the actual situation in Assen, however that the travel demand and parking durations have been assumed in a way that the authors trust is realistic, but not necessarily representative.

For this case setting, the PSR-SUE flow assignment is solved using Algorithm 1, where PSR choice sets are generated using Algorithm 2 and as network loading model we use the microsimulation traffic flow model called ITS modeler. ITS modeler is an application that runs on Paramics and is developed by TNO. It provides an advanced modeling framework for impact assessment studies of intelligent transport systems applications (for more information see Tideman and Van Noort 2013). TNO provided also the Assen road network that in earlier projects has been calibrated with respect to driving, network, and traffic control parameters. As we are dealing here with a discrete microsimulation model in combination with a probabilistic PSR choice model, travel times are computed by averaging over 10 simulation runs with different random seeds.

Overall, the dynamics of the parking search routes, traffic flows, and occupancies of parking locations appear plausible. Here we report the parking search times for a few illustrative scenarios, given in Table 4. For reference we include a base scenario considering the case as described above.

First the effect of parking reservation is tested. As expected, users of a parking reservation system will experience a lower parking search time since they are now guaranteed of never having to visit more than 1 parking location. That is, in the model drivers who use parking reservation are assigned a parking spot, which they directly route towards, without the need for any queueing or searching process.

In the simulation the mean travel time of users is reduced by about $13 \%$ compared to the reference case. The effect that this has on the travel times of the other drivers not using the parking reservation system is small and depends on the penetration rate. With a low penetration rate of $20 \%$ users the mean travel time for non-users very slightly increases, by about $1 \%$, mostly due to the extra competition for the now fewer parking spots that need to be shared among the non-users. However, with a higher penetration rate of $40 \%$ users the mean travel time for non-users slightly decreases, by about $2 \%$, due to the reduced parking search traffic between parking locations. The latter reason also causes lower variability of travel times for all drivers in all situations.

Second the effect of on-street parking is tested. To this end, we add neighborhoods with on-street parking capacity as parking 'locations' in our PSR-SUE model, and to model the on-street search process for a parking spot within such a neighborhood we 
adopt the myopic random search proposed by Kaplan and Bekhor (2011). This way, a PSR connects neighborhoods, within such a neighborhood this random search process is followed, and a driver continues to the next neighborhood (i.e. parking location) in case of not finding a vacant parking spot within the maximum admissible search time. Note that this way on-street parking and off-street parking are different processes. Regarding the physical queueing/searching process in the traffic flow simulation model, for on-street parking we employ the myopic random search model, while for off-street parking we employ the FIFO service rule at the parking facility. Regarding the expected probability of parking availability (after some queueing/searching time) in the choice model, for on-street parking we employ the SIRO queue model, while for off-street parking we employ the FIFO queue model.

In the simulation drivers opting for on-street parking experience a much higher travel time, of about $162-178 \%$ more than in the reference case, as well as a much higher standard deviation of travel time. This is largely due to the fact that the search process for on-street parking is less efficient. Note that, unlike the case for off-street parking, for on-street parking it is possible that drivers do not find a vacant parking spot within the allotted search time even though the parking location is not full (yet). At the same time, the mean travel time for off-street parking is about 7-11\% lower (and the travel time variability is lower) compared to the reference case, because a share of the drivers are now no longer using the off-street parking capacity.

Table 4. PSR-SUE model results for Assen application.

scenario individual travel time:

mean (std. dev.)

Base scenario: only off-street parking

- all

$9: 41(4: 54)$

\section{Effect of parking reservation system:}

- for $80 \%$ non-user and $20 \%$ user

- non-user

$9: 46(4: 48)$

- user

- for $60 \%$ non-user and $40 \%$ user

- non-user

- user

\section{Effect of on-street search process:}

- for 95\% off-street and 5\% on-street parking

- off-street parkers

- on-street parkers

25:25 (18:41) 
- for $85 \%$ off-street and 15\% on-street parking

- off-street parkers

- on-street parkers

26:53 (19:30)

\section{Conclusion}

In this paper we define and formulate the concept of parking search routes where a driver visits a sequence of parking locations until the first vacant parking spot is found and in doing so may account for expected parking probabilities. From there we define and formulate the stochastic user equilibrium traffic assignment in which no driver, by unilaterally changing its PSR, can lower its perceived expected generalized costs. Recognizing the interdependency between PSR flows, travel times and parking probabilities, we propose a queuing model in order to compute endogenous parking probabilities accounting for these factors as well as maximum admissible search times. To solve the PSR-SUE model we propose a solution algorithm, including a method for PSR choice set generation.

The model is implemented and applied both to a number of experimental cases to verify its properties and to a real-life setting to illustrate its usefulness in parkingrelated studies. These analyses relate mostly to the situation in which parking occupancies are fairly high. Particularly under those circumstances, there are conflicting interests from a network performance perspective. On the one hand, high parking occupancies are beneficial to ensure that parking locations are operating economically. On the other hand, high parking occupancies also cause parking search traffic. The PSR-SUE model proposed in this paper enables evaluating this trade-off under various policy scenarios and priorities.

The PSR-SUE model computes the stochastic user equilibrium traffic assignment with equilibrated parking search routes (based on random utility maximization), as a generalization of the Wardrop equilibrium concept. The assumption of stochastic equilibrium network flows implies that the model is appropriate when it can be assumed that drivers are reasonably familiar with average day-to-day travel times and parking availability conditions. In some cases, such a model assumption may be too restrictive, e.g. when day-to-day variations in the travel times and parking conditions are large and drivers receive on-trip information about these conditions. In this regard, a viable line of future research is extending the PSR-SUE model as hybrid (parking search) route choice model, combining pre-trip routing based on expected conditions and on-trip rerouting based on real-time information (see e.g., Pel et al, 2009).

The proposed PSR-SUE model yields plausible results for the various experiments and application, yet we would like to point out two remaining issues that may require further research and validation. First, the individual model components are based on well-established theories and are validated, while the overall model framework is qualitatively verified. The choice model for parking location(s) implements discrete 
choice theory based on random utility maximization, where the utility specification is calibrated and validated based on stated preference experiments by Chaniotakis and Pel (2015). The assignment model implements the theory of user equilibrium with endogenous travel and parking conditions. The parking probability model implements queueing theory where probabilities are analytically derived (and numerically solved) from statistical distributions. The overall model framework is verified via three experiments and a case study application (with calibrated traffic network and parking facilities, and realistic travel demand and parking durations). These efforts show the tractability, applicability and qualitative validity of the proposed model. Further research aimed at a more conclusive and in-depth validation of the proposed model would be recommended by using empirical observations on tracked parking search routes.

Second, the choice model adopted here for PSR flows is based on the multinomial logit model and as such assumes that attribute preferences are homogenous among drivers and that alternatives are uncorrelated in their unobserved utility. The current literature on parking location choice behavior does not provide strong evidence that these assumptions are too restrictive; also considering that the most important endogenous factors such as travel times and parking probabilities are explicitly included in the observed utility. Nevertheless, whether these assumptions are also justified for the case of parking search route choice behavior may be a subject for further research.

\section{Acknowledgements}

The authors thank Martijn van Noort at TNO, the Netherlands, for providing support with using ITS Modeler in the model application described in Section 3.2. The authors also thank three anonymous reviewers for their helpful contributions.

\section{Appendix A}

For reference with respect to denotation, Table 5 provides a list of model variables.

Table 5. Notation for model variables.

\begin{tabular}{ll}
\hline \hline Notation & Description \\
\hline$A(k)$ & cumulative arrivals at the tail of the queue until time $k$ \\
\hline$a_{\pi}\langle k, k+\kappa\rangle$ & $\begin{array}{l}\text { expected number of drivers that arrive at parking location } \pi \text { during } \\
\text { the period }\langle k, k+\kappa\rangle\end{array}$ \\
\hline$\tilde{c}_{p}(\mathbf{f})$ & stochastic costs of PSR $p$ for flow vector $\mathbf{f}$ \\
\hline$c_{p}(\mathbf{f})$ & deterministic costs of PSR $p$ for flow vector $\mathbf{f}$ \\
\hline
\end{tabular}




\begin{tabular}{|c|c|}
\hline c & number of servers (i.e. parking capacity) \\
\hline$D(k)$ & $\begin{array}{l}\text { cumulative departures from the queue (due to reneging) or from the } \\
\text { servers (after being served) until time } k\end{array}$ \\
\hline $\mathbf{f} \equiv\left[f_{p}\right]_{p \in \mathbf{P}}$ & vector of all PSR flows, where $f_{p}$ is the flow on PSR $p$ \\
\hline$\Delta F_{r s}$ & minimum flow for any used PSR for OD-pair $(r, s)$ \\
\hline$I_{p}$ & total number of parking locations included in PSR $p$ \\
\hline $\mathbf{P} \equiv\left[P_{r s}\right]$ & $\begin{array}{l}\text { vector of all PSR choice sets, where } P_{r s} \text { is the PSR choice set for } \\
\text { origin-destination pair }(r, s)\end{array}$ \\
\hline$p(i)$ & $i^{\text {th }}$ parking location for PSR $p$. \\
\hline$(p(i), p(j))$ & route segment(s) connecting $i^{\text {th }}$ and $j^{\text {th }}$ parking locations for PSR $p$ \\
\hline$S(k)$ & $\begin{array}{l}\text { cumulative number of drivers that have been or are being served } \\
\text { until time } k\end{array}$ \\
\hline$V_{\delta}(\mathbf{f})$ & utility associated with driving along route segment $\delta$ \\
\hline$V_{\pi}(\mathbf{f})$ & utility associated with parking at parking location $\pi$ \\
\hline$\beta_{c}$ & choice parameter for parking fee \\
\hline$\beta_{m}$ & choice parameter for parking type \\
\hline$\beta_{t}$ & choice parameter for travel time \\
\hline$\beta_{w}$ & choice parameter for walking distance \\
\hline$\beta_{y^{0}}$ & choice parameter for probability of parking availability upon arrival \\
\hline$\beta_{y^{\sigma}}$ & $\begin{array}{l}\text { choice parameter for probability of parking availability after search } \\
\text { time } \sigma\end{array}$ \\
\hline$\gamma_{\pi}$ & parking fee \\
\hline$\phi_{r s}$ & $\begin{array}{l}\text { minimum stochastic costs of any used PSR or costs that would be } \\
\text { experienced by a flow of } \Delta F_{r s} \text { on any currently unused PSR. }\end{array}$ \\
\hline$\Lambda$ & costs of not finding a vacant parking spot anywhere along the PSR \\
\hline$\lambda$ & step size parameter in PSR-SUE solution algorithm \\
\hline $\boldsymbol{\Psi} \equiv\left[\psi_{\pi}\right]$ & vector of parking probabilities at all parking locations $\pi$ \\
\hline$\psi_{\pi}^{0}$ & probability of parking availability upon arrival \\
\hline
\end{tabular}




\begin{tabular}{ll}
\hline$\psi_{\pi}^{\sigma}$ & probability of parking availability within search time $\sigma$ \\
\hline$\mu_{\pi}$ & parking type \\
\hline $\boldsymbol{\tau} \equiv\left[\tau_{\delta}\right]$ & vector of travel times on all route segments $\delta$ \\
\hline$\tau_{o, p(i)}$ & route travel time from origin to parking location $p(i)$ \\
\hline$\theta$ & logit scale parameter in PSR choice model \\
\hline$\omega_{\pi}$ & walking distance from parking location to destination \\
\hline \hline
\end{tabular}

\section{References}

1. Barrer, D.Y. (1957) Queuing with impatient customers and ordered service. Operations Research, 5, 650-656

2. Benenson, I., K. Martens, and S. Birfir (2008) PARKAGENT: an agent-based model of parking in the city. Computers, Environment, and Urban Systems, 32, 431-439

3. Bifulco, G.N. (1993) A stochastic user equilibrium assignment model for the evaluation of parking policies. European Journal of Operational Research, 71(2), 269-287

4. Bliemer, M.C.J., M.P.H. Raadsen, E.-S. Smits, B. Zhou, and M.G.H. Bell (2014) Quasi-dynamic traffic assignment with residual point queues incorporating a first order node model. Transportation Research Part B: Methodological, 68, 363-384

5. Bonsall, P, and I. Palmer (2004) Modelling drivers car parking behavior using data from a travel choice simulator. Transportation Research Part C: Emerging Technologies, 12(5), 321-347

6. Boyles S.D., S. Tang, and A. Unnikrishnan (2014) Dynamic traffic assignment and the parking search process. In: Proceedings of the Dynamic Traffic Assignment Symposium, Salerno, Italy.

7. Boyles S.D., S. Tang, and A. Unnikrishnan (2015) Parking search equilibrium on a network. Transportation Research Part B: Methodological, 31(2), 390-409

8. Cao J., and M. Menendez (2015) System dynamics of urban traffic based on its parking-related-states. Transportation Research Part B: Methodological, 81(3), 718-736

9. Chaniotakis, E., and A.J. Pel (2015) Drivers parking location choice under uncertain parking availability and search times: A stated preference experiment. Transportation Research Part A: Policy and Practice, 82, 228-239

10. Fiorenzo-Catalano, S. and N.J. Van der Zijpp (2001) A forecasting model for inland navigation based on route enumeration. In: Proceedings of the European Transport Conference, Cambridge, United Kingdom.

11. Geng, Y., and C.G. Cassandras (2012) A new smart parking system infrastructure and implementation. Procedia Social and Behavioral Sciences, 54, 1278-1287 
12. Geroliminis, N. (2015) Cruising-for-parking in congested cities with an MFD representation. Economics of Transportation, 4(3), 156-165

13. Guo, L., S. Huang, J. Zhuang, and A.W. Sadek (2013) Modeling parking behavior under uncertainty: A static game theoretic versus a sequential neoadditive capacity modeling approach. Networks and Spatial Economics, 13(3), 327-350

14. Kaplan, S., and S. Bekhor (2011) Exploring en-route parking type and parkingsearch route choice. In: Proceedings of the International Choice Modelling Conference, Leeds, United Kingdom.

15. Lam, W.H.K., Z.C. Li, H.J. Huang, and S.C. Wong (2006) Modeling timedependent travel choice problems in road networks with multiple user classes and multiple parking facilities. Transportation Research Part B: Methodological, 40, 368-395

16. Leurent, F., and H. Boujnah (2014) A user equilibrium, traffic assignment model of network route and parking lot choice, with search circuits and cruising flows. Transportation Research Part C: Emerging Technologies, 47(1), 28-46

17. Li, Z.C., W.H.K. Lam, H.J. Huang, and D.L. Zhu (2008) Reliability evaluation for stochastic and time-dependent networks with multiple parking facilities. Networks and Spatial Economics, 8(4), 355-381

18. Liu, W., and N. Geroliminis (2016) Modeling the morning commute for urban networks with cruising-for-parking: An MFD approach. Transportation Research Part B: Methodological, 93, 470-494

19. Liu, W., F. Zhang, and H. Yang (2016) Managing morning commute with parking space constraints in the case of a bi-modal many-to-one network. Transportmetrica A: Transport Science, 12(2), 116-141

20. Martens, K., and I. Benenson (2008) Evaluating urban parking policies with agent-based model of driver parking behavior. Transportation Research Record, 2046, 37-44

21. Movaghar, A. (1998) On queueing with customer impatience until the beginning of service. Queueing Systems, 29, 337-350

22. Pel, A.J., M.C.J. Bliemer, and S.P. Hoogendoorn (2009) Hybrid route choice modeling in dynamic traffic assignment. Transportation Research Record, 2091, 100-107

23. Shoup, D.C. (2006) Cruising for parking. Transport Policy, 13, 479-486

24. Taale, H., and A.J. Pel (2015) Better convergence for dynamic traffic assignment methods. Transportation Research Procedia, 10, 197-206

25. Tang, S., T. Rambha, R. Hatridge, S.D. Boyles, and A. Unnikrishnan (2014) Modeling parking search on a network using stochastic shortest paths with history dependence. Transportation Research Record, 2467, 73-79

26. Tideman, M., and M. Van Noort (2013) A simulation tool suite for developing connected vehicle systems. In: Proceedings of the IEEE Intelligent Vehicles Symposium, Gold Coast, Australia.

27. Van der Waerden, P. (2012) Pamela, a parking analysis model for predicting effects in local areas. $\mathrm{PhD}$ dissertation, Eindhoven University of Technology, the 
Netherlands

28. Van Ommeren, J.N., D. Wentink, and P. Rietveld (2012) Empirical evidence on cruising for parking. Transportation Research Part A: Policy and Practice, 46(1), 123-130

29. Waraich, R. A., and K.W. Axhausen (2012) Agent-based parking choice model. Transportation Research Record, 2319, 39-46

30. Waterson, B.J., N.B. Hounsell, and K. Chatterjee (2001) Quantifying the potential savings in travel time resulting from parking guidance systems - a simulation case study. Journal of the Operational Research Society, 52(10), 1067-1077

31. Watling, D.P., T.K. Rasmussen, C.G. Prato, and O.A. Nielsen (2015) Stochastic user equilibrium with equilibrated choice sets: Part I - Model formulations under alternative distributions and restrictions. Transportation Research Part B: Methodological, 77, 166-181

32. Yang, H., W. Liu, X. Wang, and X. Zhang (2013) On the morning commute problem with bottleneck congestion and parking space constraints.

Transportation Research Part B: Methodological, 58, 106-118 